\title{
Bulk-Boundary Correspondence for Non-Hermitian Hamiltonians via Green Functions
}

\author{
Heinrich-Gregor Zirnstein $\odot,{ }^{1}$ Gil Refael, ${ }^{2}$ and Bernd Rosenow ${ }^{1,3}$ \\ ${ }^{1}$ Institut für Theoretische Physik, Universität Leipzig, Brüderstrasse 16, 04103 Leipzig, Germany \\ ${ }^{2}$ Institute of Quantum Information and Matter and Department of Physics, California Institute of Technology, \\ Pasadena, California 91125, USA \\ ${ }^{3}$ Department of Condensed Matter Physics, Weizmann Institute of Science, Rehovot 76100, Israel
}

(Received 16 July 2020; accepted 4 May 2021; published 28 May 2021)

\begin{abstract}
Genuinely non-Hermitian topological phases can be realized in open systems with sufficiently strong gain and loss; in such phases, the Hamiltonian cannot be deformed into a gapped Hermitian Hamiltonian without energy bands touching each other. Comparing Green functions for periodic and open boundary conditions we find that, in general, there is no correspondence between topological invariants computed for periodic boundary conditions, and boundary eigenstates observed for open boundary conditions. Instead, we find that the non-Hermitian winding number in one dimension signals a topological phase transition in the bulk: It implies spatial growth of the bulk Green function.
\end{abstract}

DOI: 10.1103/PhysRevLett.126.216407

Topology has made a profound impact on the description and design of wavelike systems such as quantum mechanical electrons [1-5] or light interacting with matter [6-11]. The key idea is to group physical systems, each described by a gapped (insulating) Hamiltonian, into the same topological class if their Hamiltonians can be continuously deformed into each other without closing the energy gap. For Hermitian Hamiltonians, the bulk-boundary correspondence states that topological invariants for periodic boundary conditions predict the presence of boundary states for open boundary conditions [1,12-16].

Recently, non-Hermitian Hamiltonians [17-21] have attracted much attention; they describe open systems with loss (dissipation) and gain (e.g., coherent amplification in a laser) [22,23]. Extending topological methods to these systems may be particularly beneficial for the design of topological protected laser modes [24-26]. Moreover, genuinely non-Hermitian Hamiltonians, i.e., Hamiltonians that cannot be deformed to a Hermitian Hamiltonian without energy bands touching, have novel topological properties not found in Hermitian systems. They can be characterized by topological invariants different from those of Hermitian systems [27-38], but the extent of a bulk-boundary correspondence is, surprisingly, much less clear [39-50].

We consider systems in one dimensions, which are particularly interesting because not only the eigenvectors but also the eigenenergies can have a nontrivial winding number. In the case of a two-band model with chiral symmetry, the Bloch Hamiltonian is off-diagonal

$$
H(k)=\left(\begin{array}{cc}
0 & q_{+}(k) \\
q_{-}(k) & 0
\end{array}\right) .
$$

In a lattice model, the lattice spacing forces the momentum $k$ to be periodic, and the $q_{ \pm}(k)$ describe closed paths in the complex plane. For example, a non-Hermitian SuSchrieffer-Heeger (SSH) model is given by $q_{ \pm}(k)=$ $(m-1)+e^{ \pm(\gamma-i k)}$, and the paths are circles with different radii centered on the real axis [51]. The eigenvalues of the matrix $H(k)$ are distinct if neither path passes through the origin; in this case, we can assign to each path a winding number around the origin. These form the $\mathbb{Z} \times \mathbb{Z}$ topological invariant of a non-Hermitian Hamiltonian in symmetry class AIII [28]. Hermitian Hamiltonians are characterized by $q_{+}(k)=q_{-}(k)^{*}$, which forces both winding numbers to be opposites of each other; a single $\mathbb{Z}$ invariant remains [3,52,53]. Genuinely non-Hermitian phases appear whenever the two winding numbers are no longer opposites of each other [28,33]. In this case, the non-Hermitian winding number, which is the winding number of the determinant $\operatorname{det}[H(k)]$, is nonzero.

Is there a bulk-boundary correspondence for the nonHermitian winding number? To answer this, we focus on response (Green) functions, which describe experimental observables in a scattering setup. We find that the bulkboundary correspondence breaks down once the nonHermitian winding number takes a nontrivial value: When the winding number changes from zero, the bulk response starts exhibiting exponential growth in space, and since periodic systems cannot accommodate such spatial growth, they do not reflect the properties of systems with open boundaries. In this Letter, we focus on the specific example of non-Hermitian Dirac fermions to discuss the above physics, while a general proof is contained in the companion paper, Ref. [54]. Green functions are more robust objects than eigenstates, because the latter are very sensitive to boundary conditions: wave functions can become localized in the presence of an open boundary, a phenomenon referred to as non-Hermitian skin effect [39,41,55-64]. This skin effect can be observable already 
for arbitrarily small non-Hermiticity, whereas an exponential growth of the bulk Green function occurs only above a critical strength of non-Hermiticity. In a semi-infinite system with only one boundary on the other hand, right eigenfunctions can be localized at the boundary, whereas there exist no corresponding normalizable left eigenfunctions, such that the Green function cannot be expressed in terms of eigenfunctions.

Example: Dirac fermions with non-Hermitian terms.We consider a continuum model that corresponds to the long distance limit of the non-Hermitian SSH model $[32,41,65,66]$. It concerns wave functions with two components $\psi(x)=\left[\psi_{1}(x), \psi_{2}(x)\right]^{T}$ subject to a Hermitian Dirac Hamiltonian $H_{0}=m \sigma_{x}+\left(-i \partial_{x}\right) \sigma_{y}$, where $\sigma_{x}, \sigma_{y}$ are Pauli matrices, $m$ is a real mass parameter (band gap). Let us introduce non-Hermiticity by adding constant antiHermitian terms:

$$
\hat{H}=\hat{H}_{0}+i \gamma \sigma_{y},
$$

where $\gamma$ is real. There are three more terms that we could add: $i \gamma_{x} \sigma_{x}, i \gamma_{z} \sigma_{z}$, and $-i \Gamma \mathbb{1}$, where $\mathbb{1}$ is the identity matrix. The first can be absorbed by analytic continuation of the mass $m$. The second and third vanish if we also impose a chiral symmetry $\left\{\hat{H}, \sigma_{z}\right\}=0$, necessary for discussing zero energy boundary eigenstates in one dimension. Thus, the symmetry class is AIII [1] for complex $m$. For real mass $m$, $\hat{H}$ is additionally invariant under complex conjugation, placing it in symmetry class BDI, which also implies that eigenvalues occur in complex conjugate pairs.

In the continuum model (2), we have $q_{ \pm}(k)=$ $m \pm(\gamma-i k)$, and the paths described by $q_{ \pm}(k)$ in the complex plane are no longer closed. Still, one can assign a half-integer winding number $\nu_{ \pm}$[33] that changes whenever a path crosses the origin. Such crossings happen at $\gamma= \pm m$ and we find the topological phase diagram in Fig. 1(a).

Open boundary conditions. - We now consider a system of length $L$ with open boundary conditions

$$
\psi_{2}(0)=0, \quad \psi_{1}(L)=0
$$

corresponding to a particular boundary termination of the lattice model. For open boundary conditions, the nonHermitian terms in both the Dirac-Hamiltonian Eq. (2) and the non-Hermitian SSH model defined below Eq. (1) can be eliminated by a similarity transformation: if $\psi_{0}(x)$ is an eigenfunction of the Hamiltonian $\hat{H}_{0}$, then $\psi(x)=$ $e^{\gamma x} \psi_{0}(x)$ is an eigenfunction of the Hamiltonian $\hat{H}$. From this we see that all eigenfunctions are exponentially localized, when $\gamma \neq 0$. This is the non-Hermitian skin effect [39,41,55-64].

Bulk and boundary Green function.-To clearly distinguish bulk and boundary, we now focus on Green functions, which are matrix-valued solutions to the equation
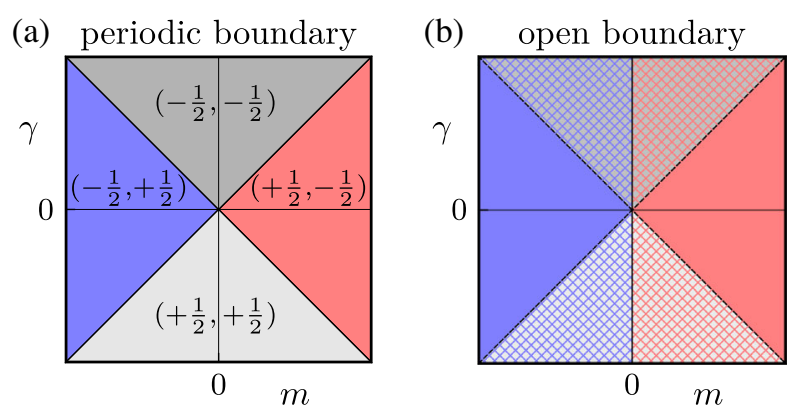

FIG. 1. Topological phase diagram of one-dimensional nonHermitian Dirac fermions with particle-hole symmetry and chiral symmetry. (a) Periodic boundary conditions. A pair of halfinteger winding numbers $\left(\nu_{+}, \nu_{-}\right)$distinguishes four phases: Two Hermitian (red and blue) and two genuinely non-Hermitian phases (gray), separated by lines $\gamma= \pm m$. (b) Open boundary conditions. The line $m=0$ separates phases with a different number of zero energy boundary eigenstates. For the boundary conditions (3), a positive mass implies the existence of a boundary state at each end (red), which are absent for a negative mass (blue). The lines $\gamma= \pm m$ now indicate that the bulk (and boundary) Green function change from exponential decay to exponential growth.

$$
(E-\hat{H}) G(E ; x, y)=1 \delta(x-y) .
$$

The bulk Green function $G_{\text {bulk }}$ is defined as the response of an infinite system [54], whereas the Green function $G_{\text {open }}$ for open boundary conditions is defined as the solution that satisfies the conditions (3). When we probe the system far away from the boundary, $0 \ll x, y \ll L$, then only the bulk of the system responds, and we expect that both Green functions give the same result. However, when the source is close to the boundary, $y \approx 0$ or $y \approx L$, we expect that reflection at the boundary is important, which is captured in the boundary Green function

$$
G_{\text {bound }}(E ; x, y):=G_{\text {open }}(E ; x, y)-G_{\text {bulk }}(E ; x-y) \text {. }
$$

It solves the homogeneous equation $(E-\hat{H})$ $G_{\text {bound }}(E ; x, y)=0$. We have used that for a translationally invariant Hamiltonian, the bulk response only depends on the difference $x-y$. If $G_{0}$ denotes a Green function of $\hat{H}_{0}$ for open boundaries, then the corresponding retarded Green function for $\hat{H}$ reads

$$
G(E ; x, y)=G_{0}(E+i \eta ; x, y) e^{\gamma(x-y)},
$$

with $\eta=0^{+}$. We now focus on zero energy, $E=0$. Then, we find

$G_{0, \text { bulk }}(i \eta ; x, y)=\left[\theta(-\tilde{x}) G_{L}+\theta(\tilde{x}) G_{R}\right] e^{-\sqrt{m^{2}+\eta^{2}}|\tilde{x}|}$,

where $\tilde{x}=x-y$, and $G_{L}$ and $G_{R}$ are matrices 


$$
G_{s}=\mathcal{N}\left(\begin{array}{cc}
i \eta & m+\nu_{s} \sqrt{m^{2}+\eta^{2}} \\
m-\nu_{s} \sqrt{m^{2}+\eta^{2}} & i \eta
\end{array}\right),
$$

with $s=L, R, \nu_{R / L}= \pm 1$, and $\mathcal{N}=1 /\left(2 \sqrt{m^{2}+\eta^{2}}\right)$. Thus, we obtain one of our main results: in the phases where the non-Hermitian winding number is nonzero, $|\gamma|>|m|$, the bulk Green function $G_{\text {bulk }}$ grows exponentially as $x \rightarrow \pm \infty$ while keeping $y$ fixed. For $G_{0 \text {,bound }}$ near the left boundary, we find

$G_{0, \text { bound }}(i \eta, x, y)=G_{B} e^{-\sqrt{m^{2}+\eta^{2}}(x+y)}, \quad$ for $x, y \ll L$

Here, $G_{B}$ is the matrix

$$
G_{B}=-G_{R}\left(\begin{array}{cc}
\frac{m+\sqrt{m^{2}+\eta^{2}}}{m-\sqrt{m^{2}+\eta^{2}}} & 0 \\
0 & 1
\end{array}\right)
$$

Taken together, this yields the decomposition (5).

Boundary eigenstates.-The Green function can be expressed as a sum over eigenstates

$$
G(E ; x, y)=\sum_{n}\left(E-E_{n}\right)^{-1}\left\langle x \mid \psi_{R}^{n}\right\rangle\left\langle\psi_{L}^{n} \mid y\right\rangle .
$$

Here, $\left|\psi_{R}^{n}\right\rangle$ are the so-called right and $\left|\psi_{L}^{n}\right\rangle$ the left eigenstates of the non-Hermitian Hamiltonian, i.e., $H\left|\psi_{R}^{n}\right\rangle=E_{n}\left|\psi_{R}^{n}\right\rangle$ and $H^{\dagger}\left|\psi_{L}^{n}\right\rangle=E_{n}^{*}\left|\psi_{L}^{n}\right\rangle$, with $\left\langle\psi_{L}^{n} \mid \psi_{R}^{m}\right\rangle=$ $\delta_{n m}$ [67]. The contribution $\left\langle x \mid \psi_{R}^{n}\right\rangle\left\langle\psi_{L}^{n} \mid y\right\rangle$ of an individual eigenstate to the Green function can be extracted as the residue of the pole at $E=E_{n}[68,69]$. For identical positions $x=y$, this residue yields the biorthogonal polarization discussed in Ref. [40]. We now define a boundary eigenstate to be the residue of a pole of the boundary Green function, and focus on states at zero energy, $E=0$. For open boundary conditions, our model has only real eigenvalues due to the relation Eq. (6), and we can obtain the residue from the imaginary part of the Green function since $\operatorname{Im} G\left(E+i 0^{+} ; x, y\right)=-\sum_{n}\left\langle x \mid \psi_{R}\right\rangle\left\langle\psi_{L} \mid y\right\rangle \delta\left(E-E_{n}\right) \quad$ for real $E$. We find that

$$
\begin{gathered}
-\operatorname{Im} G^{11}{ }_{\text {bound }}(0, x, y)=A e^{(\gamma-m) x} e^{(-\gamma-m) y}, \\
\text { where } A=\theta(m) 2 m / \eta \quad \text { with } \quad \eta=0^{+} .
\end{gathered}
$$

Thus, for $m>0$, the boundary Green function has an isolated pole at zero energy, whose associated eigenstate is $\left\langle x \mid \psi_{R}^{0}\right\rangle=e^{(\gamma-m) x}$ and $\left\langle\psi_{L}^{0} \mid y\right\rangle=e^{(-\gamma-m) y}$. The spatial shape changes dramatically from exponentially localized to exponentially growing and vice versa whenever $\gamma= \pm m$. In contrast, for $m<0$, no boundary eigenstate is found. Thus, the number of zero energy boundary eigenstates does not change during the topological phase transition at $\gamma= \pm|m|$ for periodic boundary conditions [Fig. 1(b)], and the bulk-boundary correspondence breaks down.
Bulk-periodic correspondence.-The traditional view on the bulk-boundary correspondence actually comprises two separate logical steps: it relates (i) the bulk to the boundary Green function, and (ii) the Green function $G_{\text {period }}$ for periodic boundary conditions to that for the bulk of an infinite system: in the limit of large system size, both agree if the bulk Green function decays spatially [Fig. 2(a)]; this allows us to use topological invariants of the Bloch Hamiltonian (1) to characterize an infinite bulk. In nonHermitian systems, step (i) is unproblematic, but step (ii) may fail. To better distinguish them, we propose to narrow the name bulk-boundary correspondence to refer only to the first step, and to call the second step the bulkperiodic correspondence.

Indeed, for our model in the regime $|\gamma|>|m|$, the bulkperiodic correspondence breaks down, because the periodic Green function decays, while the bulk Green function grows exponentially [Fig. 2(b)]. This growth also explains the exponential sensitivity to small perturbations seen in Ref. [44]. For periodic boundary conditions $\psi(-L / 2)=$ $\psi(+L / 2)$, and using the results Eqs. (6) and (7) for the bulk Green function, we find

$$
\begin{aligned}
G_{\text {period }}(0 ; x, 0)= & G_{\text {bulk }}(0 ; x, 0)+G_{L} \frac{e^{\kappa_{L} x}}{e^{\kappa_{L} L}-1} \\
& +G_{R} \frac{e^{\kappa_{R} x}}{e^{-\kappa_{R} L}-1},
\end{aligned}
$$

with $\kappa_{L / R}=\gamma \pm \sqrt{m^{2}+\eta^{2}}$. In the limit of large system size $L \gg|x|$, the two additional terms vanish if only if the

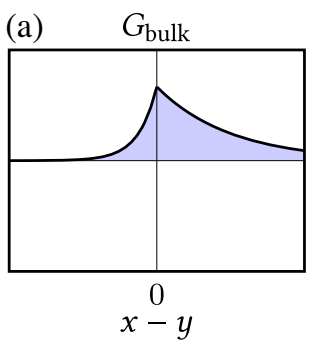

(b)

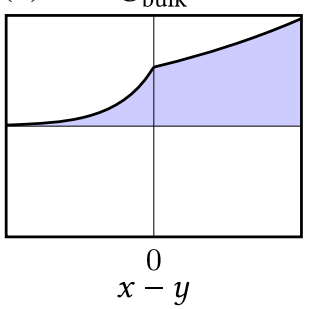

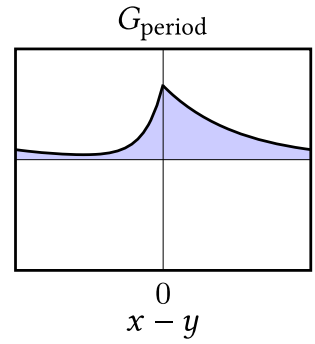

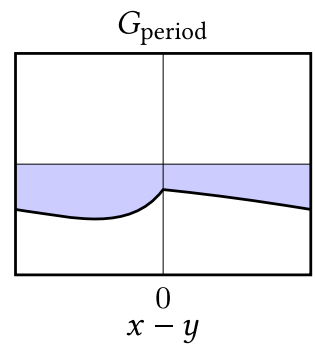

FIG. 2. Breakdown of the bulk-periodic correspondence. (a) If the bulk Green function decays spatially, then both bulk and periodic Green function agree. (b) If the bulk Green function grows spatially, then the periodic Green function has to change drastically in order to accommodate periodic boundary conditions. 
exponents satisfy $\kappa_{L}>0$ and $\kappa_{R}<0$, i.e., if the bulk Green function decays spatially [Fig. 2].

If we focus on bulk growth and disregard boundary eigenstates, we no longer require symmetry class AIII. Then, we find our main result, which holds both with and without symmetry (class A): if the non-Hermitian winding number is nonzero, then the bulk Green function at zero energy grows spatially. For example, consider a general Dirac model $\hat{H}=\left(-i \partial_{x}\right) \tau_{1}+m_{1} \tau_{1}+m_{2} \tau_{2}+\cdots+$ $m_{n} \tau_{n}-i \Gamma \mathbb{1}$ where the $\tau_{j}$ are Hermitian gamma matrices $\left\{\tau_{i}, \tau_{j}\right\}=2 \delta_{i j}$, the masses $m_{j}$ are complex and $\Gamma \geq 0$. Then, since $(\hat{H}+i \Gamma)^{2}$ is proportional to the identity matrix, the corresponding bulk Green function has form $G_{\text {bulk }}(0 ; x, 0)=G_{L} \theta(-x) e^{\kappa_{L} x}+G_{R} \theta(x) e^{\kappa_{R} x}$ with $\kappa_{L, R}=$ $-i m_{1} \pm \sqrt{m_{2}^{2}+\cdots+m_{n}^{2}+\Gamma^{2}}$ and appropriate $G_{L, R}$. Here, the branch of the complex square root is the one with positive real part; we choose it by demanding that we remain in the same branch when $\Gamma \rightarrow \infty$ [54]. Thus, the Green function grows in space if and only if the imaginary part of $m_{1}$ exceeds the real part of the root. Imposing symmetries will only constrain the parameters, but not affect this conclusion. In general, the Green function is a sum of exponentials $\exp \left(i k_{s} x\right)$ where the complex momenta $k_{s}$ are the zeros of $\operatorname{det}[H(k)]$; the subscript $s$ refers to a side $L, R$, and an index. For local continuum models like the Dirac model, this determinant is a polynomial in momentum $k$. Thus, the non-Hermitian winding number $\nu(H)=(2 \pi)^{-1} \int_{-\infty}^{\infty} d k \partial_{k} \arg \operatorname{det}[H(k)]$ is the sum of $+1 / 2$ for each zero above the real axis and $-1 / 2$ for each zero below. But this number changes precisely when one of the zeros crosses the real axis, which means that the exponential changes from spatial decay to spatial growth. We extend this sketch to a full proof for lattice models in Ref. [54]. While for the above Dirac models, exponential growth only occurs in the genuinely non-Hermitian phases, for other models, it may arise even when the Hamiltonian can be deformed to a Hermitian one; see [51] for an example. Thus, the growth of the bulk Green function is not, by itself, topologically invariant.

Our work still leaves open the exciting question of the bulk-boundary correspondence in the narrow sense: are there topological invariants of the bulk Green function that imply the presence of boundary eigenstates? For Dirac fermions, the latter persist well into the genuinely nonHermitian phases. This is also true for lattice models discussed in the literature [32,39-42,44]; see the Supplemental Material [51] for details.

Experimental response: Scattering.-In a scattering configuration [see Fig. 3(a)], an excitation of an outside field $\phi$ is created, and the incoming amplitude $\phi^{-}$targets a point $x_{1}$ of the system. If the excitation is monochromatic with frequency (energy) $E$, then the system will eventually reach a stationary state $\psi$ that, in turn, emits an outgoing amplitude $\phi^{+}$at every position $x_{2}$. The scattering matrix
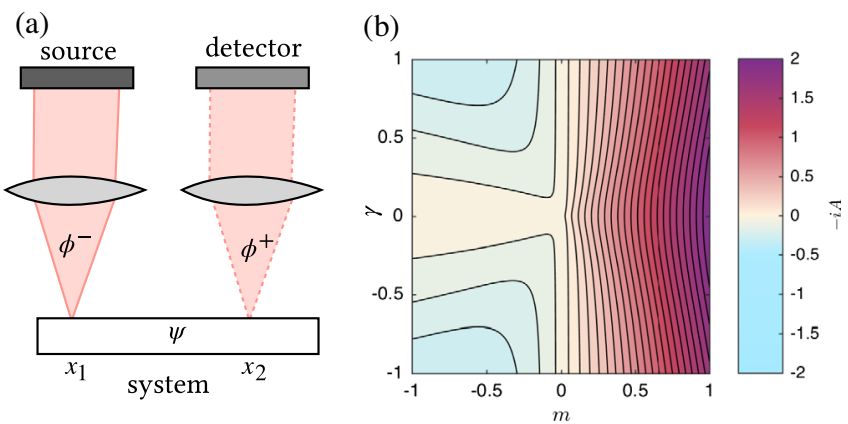

FIG. 3. (a) Scattering setup. (b) Scattering response of the onedimensional Dirac fermion with dissipation $\Gamma=|\gamma|$. The plot shows the amplitude $-i A$ of the boundary response at the left boundary.

$S(E)$ records how incoming amplitudes are mapped to outgoing amplitudes, $\phi^{+}=S(E) \phi^{-}$, and is given by the Mahaux-Weidenmüller formula [70]

$S(E)=\mathbb{1}-2 i W^{\dagger} \frac{1}{E-\hat{H}} W, \quad \hat{H}=\hat{H}_{0}-i W W^{\dagger}$.

Here, the matrix $W$ describes how the outside field couples into the system. The Hamiltonian $\hat{H}_{0}$ describes the time evolution of the system if the coupling was absent; it is usually Hermitian, for it is the combination with the dissipative term $-i W W^{\dagger}$ that yields an effective nonHermitian Hamiltonian $\hat{H}$. The scattering matrix differs from unity by $-2 i W^{\dagger} G(E) W$ where $G(E)$ is the Green function of this non-Hermitian Hamiltonian. To make this Letter self-contained, in the Supplemental Material [51] we include an elementary discussion of the MahauxWeidenmüller formula [71,72], which is equivalent to temporal coupled mode theory $[73,74]$ in optics.

So far, we have shown how coupling to an environment yields a non-Hermitian Hamiltonian. Conversely, we now consider a Hamiltonian $H$ and attempt to realize it in a scattering setup. For this, we decompose it as $\hat{H}=\hat{H}_{0}+i \hat{\Gamma}$ where $\hat{H}_{0}=\left[\hat{H}+\hat{H}^{\dagger}\right] / 2$ and $\hat{\Gamma}=(i / 2)\left[\hat{H}^{\dagger}-\hat{H}\right]$ are Hermitian matrices. We define a Hamiltonian to be purely dissipative if $\hat{\Gamma}$ is negative semidefinite, $\hat{\Gamma} \leq 0$, i.e., if it has no positive eigenvalues. Any matrix of the form $\hat{\Gamma}=$ $-W W^{\dagger}$ is negative semidefinite, and any semidefinite $\hat{\Gamma}$ can be represented in the above form by choosing $W=(-\hat{\Gamma})^{1 / 2}$. This choice is unique up to a change of basis for the outside field, $W \rightarrow W U$, with $U$ unitary, and up to components that do not couple. Thus, any purely dissipative Hamiltonian can be realized in a scattering setup described by Eq. (14). But in order to realize a general nonHermitian Hamiltonian, we have to allow for positive eigenvalues in the anti-Hermitian part, which corresponds to (linear) gain, i.e., coherent amplification. In fact, gain is a key requirement for the non-Hermitian winding number to be nonzero, see Ref. [54]. 
In optical systems, gain can be realized by optical pumping [75], and spatially uniform gain can be described by the ansatz $\hat{H}=\hat{H}_{0}+i g-i W W^{\dagger}$ with a positive constant $g$ in Eq. (14). Then, the anti-Hermitian part of $\hat{H}$ is related to the coupling matrix via $-W W^{\dagger}=\hat{\Gamma}-g$, and this relation can be solved for $W$ if the non-Hermitian Hamiltonian $\hat{H}$ has bounded gain, i.e., if $\hat{\Gamma}-g$ becomes negative semidefinite by making $g$ sufficiently large. For example, the Dirac fermion, Eq. (2), can be described by $\hat{H}=\hat{H}_{0}+i g-i W W^{\dagger}$ with $g=|\gamma|, \quad W=\sqrt{|\gamma|}[1$, $-\operatorname{sgn}(\gamma) i]^{T}$; the coupling $W$ is a $2 \times 1$ matrix when modeling the outside field as a scalar.

Since a purely dissipative setup can be realized more easily than one with gain, we ask whether signatures of the zero energy boundary states of the Dirac fermion Eq. (2) can still be found without gain. This corresponds to allowing a constant dissipation, $\hat{H}=\hat{H}_{0}+i \gamma \sigma_{y}-i \Gamma \mathbb{1}$, which does break chiral symmetry. We consider the special case $\Gamma=|\gamma|$, which has the least amount of dissipation while probing the phase transition region $\gamma= \pm|m|$. At zero energy, we can use Eq. (9) with $\eta=\Gamma$ and find

$$
\begin{aligned}
& {\left[W^{\dagger} G_{\text {bound }}(E=0) W\right]\left(x_{2}, x_{1}=0\right)=A \exp \left(-x_{2} / \xi_{L}\right),} \\
& A=-i \frac{m}{\sqrt{m^{2}+\gamma^{2}}} \frac{\gamma^{2}}{m-\sqrt{m^{2}+\gamma^{2}}},
\end{aligned}
$$

where $A$ denotes the amplitude and $\xi_{L}=1 /\left(\sqrt{m^{2}+\gamma^{2}}-\right.$ $\gamma)$ the localization length of the response. We see that the amplitude can still distinguish the phase with boundary eigenstate, $m>0$, from the phase without, $m<0$, though the distinction is less sharp [Fig. 3(b)] than in the case with gain and chiral symmetry.

Conclusion.-Using the example of non-Hermitian Dirac fermions, we have discussed the topological phase diagram via Green functions. The key idea was to decompose the Green function for open boundary conditions into a bulk and a boundary Green function, Eq. (5). We have shown that a nonzero non-Hermitian winding number means that the correspondence of the open system to a periodic one breaks down due to an exponential growth of the bulk Green function. We have also discussed how to realize non-Hermitian Hamiltonian in a scattering setup and indicated that observing a nonzero topological invariant requires a system with gain.

We would like to thank T. Karzig for helpful discussions. B. R. and H.-G. Z. acknowledge financial support from the German Research Foundation within the CRC 762 (project B6). B. R. acknowledges support from the Rosi and Max Varon Visiting Professorship at the Weizmann Institute of Science. We are grateful for the hospitality of the Aspen Center for Physics, funded by NSF Grant No. PHY1607611, where part of this work was performed. G. R. is grateful for generous support from the Institute of
Quantum Information and Matter, an NSF frontier center, NSF Grant No. 1839271, and The Simons Foundation.

[1] M.Z. Hasan and C. L. Kane, Colloquium: Topological insulators, Rev. Mod. Phys. 82, 3045 (2010).

[2] X.-L. Qi and S.-C. Zhang, Topological insulators and superconductors, Rev. Mod. Phys. 83, 1057 (2011).

[3] C.-K. Chiu, J. C. Y. Teo, A. P. Schnyder, and S. Ryu, Classification of topological quantum matter with symmetries, Rev. Mod. Phys. 88, 035005 (2016).

[4] B. Andrei Bernevig and T. L. Hughes, Topological Insulators and Topological Superconductors (Princeton University Press, Princeton, 2013).

[5] A. Bansil, H. Lin, and T. Das, Colloquium: Topological band theory, Rev. Mod. Phys. 88, 021004 (2016).

[6] S. Raghu and F. D. M. Haldane, Analogs of quantum-Halleffect edge states in photonic crystals, Phys. Rev. A 78, 033834 (2008).

[7] L. Lu, J. D. Joannopoulos, and M. Soljačić, Topological photonics, Nat. Photonics 8, 821 (2014).

[8] A. B. Khanikaev and G. Shvets, Two-dimensional topological photonics, Nat. Photonics 11, 763 (2017).

[9] M. C. Rechtsman, J. M. Zeuner, Y. Plotnik, Y. Lumer, D. Podolsky, F. Dreisow, S. Nolte, M. Segev, and A. Szameit, Photonic Floquet topological insulators, Nature (London) 496, 196 (2013).

[10] Y. Plotnik, M. C. Rechtsman, D. Song, M. Heinrich, J. M. Zeuner, S. Nolte, Y. Lumer, N. Malkova, J. Xu, A. Szameit, Z. Chen, and M. Segev, Observation of unconventional edge states in 'photonic graphene,' Nat. Mater. 13, 57 (2014).

[11] J. Noh, W. A. Benalcazar, S. Huang, M. J. Collins, K. P. Chen, T. L. Hughes, and M. C. Rechtsman, Topological protection of photonic mid-gap defect modes, Nat. Photonics 12, 408 (2018).

[12] B. I. Halperin, Quantized Hall conductance, current-carrying edge states, and the existence of extended states in a two-dimensional disordered potential, Phys. Rev. B 25, 2185 (1982).

[13] Y. Hatsugai, Chern Number and Edge States in the Integer Quantum Hall Effect, Phys. Rev. Lett. 71, 3697 (1993).

[14] A. M. Essin and V. Gurarie, Bulk-boundary correspondence of topological insulators from their respective Green's functions, Phys. Rev. B 84, 125132 (2011).

[15] G. M. Graf and M. Porta, Bulk-edge correspondence for two-dimensional topological insulators, Commun. Math. Phys. 324, 851 (2013).

[16] J. C. Avila, H. Schulz-Baldes, and C. Villegas-Blas, Topological invariants of edge states for periodic two-dimensional models, Math. Phys. Anal. Geom. 16, 137 (2013).

[17] N. Hatano and D. R. Nelson, Localization Transitions in Non-Hermitian Quantum Mechanics, Phys. Rev. Lett. 77, 570 (1996).

[18] C. M. Bender and S. Boettcher, Real Spectra in NonHermitian Hamiltonians Having PT Symmetry, Phys. Rev. Lett. 80, 5243 (1998).

[19] I. Rotter, A non-Hermitian Hamilton operator and the physics of open quantum systems, J. Phys. A 42, 153001 (2009). 
[20] H. Cao and J. Wiersig, Dielectric microcavities: Model systems for wave chaos and non-Hermitian physics, Rev. Mod. Phys. 87, 61 (2015).

[21] B. Zhen, C. W. Hsu, Y. Igarashi, L. Lu, I. Kaminer, A. Pick, S.-L. Chua, J. D. Joannopoulos, and M. Soljačic, Spawning rings of exceptional points out of Dirac cones, Nature (London) 525, 354 (2015).

[22] S. Longhi, Parity-time symmetry meets photonics: A new twist in non-Hermitian optics, Europhys. Lett. 120, 64001 (2017).

[23] R. El-Ganainy, K. G. Makris, M. Khajavikhan, Z. H. Musslimani, S. Rotter, and D. N. Christodoulides, Non-Hermitian physics and PT symmetry, Nat. Phys. 14, 11 (2018).

[24] B. Bahari, A. Ndao, F. Vallini, A. El Amili, Y. Fainman, and B. Kanté, Nonreciprocal lasing in topological cavities of arbitrary geometries, Science 358, 636 (2017).

[25] P. St-Jean, V. Goblot, E. Galopin, A. Lemaître, T. Ozawa, L. Le Gratiet, I. Sagnes, J. Bloch, and A. Amo, Lasing in topological edge states of a one-dimensional lattice, Nat. Photonics 11, 651 (2017).

[26] M. Parto, S. Wittek, H. Hodaei, G. Harari, M. A. Bandres, J. Ren, M. C. Rechtsman, M. Segev, D. N. Christodoulides, and M. Khajavikhan, Edge-Mode Lasing in 1D Topological Active Arrays, Phys. Rev. Lett. 120, 113901 (2018).

[27] E. J. Bergholtz, J. C. Budich, and F. K. Kunst, Exceptional topology of non-Hermitian systems, Rev. Mod. Phys. 93, 015005 (2021).

[28] Z. Gong, Y. Ashida, K. Kawabata, K. Takasan, S. Higashikawa, and M. Ueda, Topological Phases of Non-Hermitian Systems, Phys. Rev. X 8, 031079 (2018).

[29] K. Kawabata, K. Shiozaki, M. Ueda, and M. Sato, Symmetry and Topology in Non-Hermitian Physics, Phys. Rev. X 9, 041015 (2019).

[30] H. Zhou and J. Y. Lee, Periodic table for topological bands with non-Hermitian symmetries, Phys. Rev. B 99, 235112 (2019).

[31] N. M. Shnerb and D. R. Nelson, Winding Numbers, Complex Currents, and Non-Hermitian Localization, Phys. Rev. Lett. 80, 5172 (1998).

[32] T.E. Lee, Anomalous Edge State in a Non-Hermitian Lattice, Phys. Rev. Lett. 116, 133903 (2016).

[33] D. Leykam, K. Y. Bliokh, C. Huang, Y. D. Chong, and F. Nori, Edge Modes, Degeneracies, and Topological Numbers in Non-Hermitian Systems, Phys. Rev. Lett. 118, 040401 (2017).

[34] H. Shen, B. Zhen, and L. Fu, Topological Band Theory for Non-Hermitian Hamiltonians, Phys. Rev. Lett. 120, 146402 (2018).

[35] S. Lieu, Topological symmetry classes for non-Hermitian models and connections to the bosonic Bogoliubov-de Gennes equation, Phys. Rev. B 98, 115135 (2018).

[36] M. R. Hirsbrunner, T. M. Philip, and M. J. Gilbert, Topology and observables of the non-Hermitian Chern insulator, Phys. Rev. B 100, 081104(R) (2019).

[37] S. Longhi, Topological Phase Transition in non-Hermitian Quasicrystals, Phys. Rev. Lett. 122, 237601 (2019).

[38] Y. Chen and H. Zhai, Hall conductance of a non-Hermitian Chern insulator, Phys. Rev. B 98, 245130 (2018).
[39] Y. Xiong, Why does bulk boundary correspondence fail in some non-Hermitian topological models, J. Phys. Commun. 2, 035043 (2018).

[40] F. K Kunst, E. Edvardsson, J. C. Budich, and E. J. Bergholtz, Biorthogonal Bulk-Boundary Correspondence in Non-Hermitian Systems, Phys. Rev. Lett. 121, 026808 (2018).

[41] S. Yao and Z. Wang, Edge States and Topological Invariants of Non-Hermitian Systems, Phys. Rev. Lett. 121, 086803 (2018).

[42] C. H. Lee and R. Thomale, Anatomy of skin modes and topology in non-Hermitian systems, Phys. Rev. B 99, 201103(R) (2019).

[43] L. Jin and Z. Song, Bulk-boundary correspondence in a nonHermitian system in one dimension with chiral inversion symmetry, Phys. Rev. B 99, 081103(R) (2019).

[44] L. Herviou, J. H. Bardarson, and N. Regnault, Defining a bulk-edge correspondence for non-Hermitian Hamiltonians via singular-value decomposition, Phys. Rev. A 99, 052118 (2019).

[45] Z.-Y. Ge, Y.-R. Zhang, T. Liu, S.-W. Li, H. Fan, and F. Nori, Topological band theory for non-Hermitian systems from the Dirac equation, Phys. Rev. B 100, 054105 (2019).

[46] D. S. Borgnia, A. J. Kruchkov, and R.-J. Slager, NonHermitian Boundary Modes and Topology, Phys. Rev. Lett. 124, 056802 (2020).

[47] W. Brzezicki and T. Hyart, Hidden Chern number in onedimensional non-Hermitian chiral-symmetric systems, Phys. Rev. B 100, 161105(R) (2019).

[48] K. Yokomizo and S. Murakami, Non-Bloch Band Theory of Non-Hermitian Systems, Phys. Rev. Lett. 123, 066404 (2019).

[49] K. Kawabata, N. Okuma, and M. Sato, Non-Bloch band theory of non-Hermitian Hamiltonians in the symplectic class, Phys. Rev. B 101, 195147 (2020).

[50] Z. Yang, K. Zhang, C. Fang, and J. Hu, Non-Hermitian Bulk-Boundary Correspondence and Auxiliary Generalized Brillouin Zone Theory, Phys. Rev. Lett. 125, 226402 (2020).

[51] See Supplemental Material at http://link.aps.org/ supplemental/10.1103/PhysRevLett.126.216407 for nonHermitian extensions of the SSH lattice model and an elementary discussion of the Mahaux-Weidenmüller formula.

[52] A. P. Schnyder, S. Ryu, A. Furusaki, and A. W. W. Ludwig, Classification of topological insulators and superconductors in three spatial dimensions, Phys. Rev. B 78, 195125 (2008).

[53] A. Kitaev, Periodic table for topological insulators and superconductors, AIP Conf. Proc. 1134, 22 (2009).

[54] H.-G. Zirnstein and B. Rosenow, companion paper, Exponentially growing bulk Green functions as signature of nontrivial non-Hermitian winding number in one dimension, Phys. Rev. B 103, 195157 (2021).

[55] V. M. Martinez Alvarez, J. E. Barrios Vargas, and L. E. F. Foa Torres, Non-Hermitian robust edge states in one dimension: Anomalous localization and eigenspace condensation at exceptional points, Phys. Rev. B 97, 121401(R) (2018). 
[56] V. M. Martinez Alvarez, J. E. Barrios Vargas, M. Berdakin, and L. E. F. Foa Torres, Topological states of non-Hermitian systems, Eur. Phys. J. Spec. Top. 227, 1295 (2018).

[57] K. Zhang, Z. Yang, and C. Fang, Correspondence between Winding Numbers and Skin Modes in Non-Hermitian Systems, Phys. Rev. Lett. 125, 126402 (2020).

[58] N. Okuma, K. Kawabata, K. Shiozaki, and M. Sato, Topological Origin of Non-Hermitian Skin Effects, Phys. Rev. Lett. 124, 086801 (2020).

[59] N. Okuma and Masatoshi Sato, Hermitian zero modes protected by nonnormality: Application of pseudospectra, Phys. Rev. B 102, 014203 (2020).

[60] L. Xiao, T. Deng, K. Wang, G. Zhu, Z. Wang, W. Yi, and P. Xue, Non-Hermitian bulk-boundary correspondence in quantum dynamics, Nat. Phys. 16, 761 (2020).

[61] T. Helbig, T. Hofmann, S. Imhof, M. Abdelghany, T. Kiessling, L. W. Molenkamp, C. H. Lee, A. Szameit, M. Greiter, and R. Thomale, Generalized bulk-boundary correspondence in non-Hermitian topolectrical circuits, Nat. Phys. 16, 747 (2020).

[62] T. Hofmann, T. Helbig, F. Schindler, N. Salgo, M. Brzezińska, M. Greiter, T. Kiessling, D. Wolf, A. Vollhardt, A. Kabaši, C. H. Lee, A. Bilušić, R. Thomale, and T. Neupert, Reciprocal skin effect and its realization in a topolectrical circuit, Phys. Rev. Research 2, 023265 (2020).

[63] T. Yoshida, T. Mizoguchi, and Y. Hatsugai, Mirror skin effect and its electric circuit simulation, Phys. Rev. Research 2, 022062(R) (2020).

[64] S. Longhi, Probing non-Hermitian skin effect and nonBloch phase transitions, Phys. Rev. Research 1, 023013 (2019).
[65] S. Lieu, Topological phases in the non-Hermitian SuSchrieffer-Heeger model, Phys. Rev. B 97, 045106 (2018).

[66] K. Esaki, M. Sato, K. Hasebe, and M. Kohmoto, Edge states and topological phases in non-Hermitian systems, Phys. Rev. B 84, 205128 (2011).

[67] D. C. Brody, Biorthogonal quantum mechanics, J. Phys. A 47, 035305 (2014).

[68] R. E. Peierls, Complex Eigenvalues in Scattering Theory, Proc. R. Soc. Math. Phys. Eng. Sci. 253, 16 (1959).

[69] M. Zworski, Resonances in physics and geometry, Not. Am. Math. Soc. 46, 319 (1999).

[70] C. Mahaux and H. A. Weidenmüller, Shell-Model Approach to Nuclear Reactions (North-Holland Pub. Co., Amsterdam, 1969).

[71] M. S. Livsic, Operators, Oscillations, Waves. (Open Systems) (American Mathematical Society, Providence, 1973).

[72] Y. V. Fyodorov and H. J. Sommers, Spectra of random contractions and scattering theory for discrete-time systems, J. Exp. Theor. Phys. Lett. 72, 422 (2000).

[73] W. Suh, Z. Wang, and S. Fan, Temporal coupled-mode theory and the presence of non-orthogonal modes in lossless multimode cavities, IEEE J. Quantum Electron. 40, 1511 (2004).

[74] S. Fan, W. Suh, and J. D. Joannopoulos, Temporal coupledmode theory for the Fano resonance in optical resonators, Opt. Soc. Am. J. 20, 569 (2003).

[75] Exponential amplification cannot be indefinite; in practice, gain will saturate nonlinearly [76].

[76] S. Malzard, E. Cancellieri, and H. Schomerus, Topological dynamics and excitations in lasers and condensates with saturable gain or loss, Opt. Express 26, 22506 (2018). 